\title{
ontome \\ pubs.acs.org/jpr

\section{Ferritin Heavy Chain Is the Host Factor Responsible for HCV-Induced Inhibition of apoB-100 Production and Is Required for Efficient Viral Infection}

Carmine Mancone, ${ }^{\dagger, \dagger}$ Claudia Montaldo, ${ }^{\dagger, \S, \ddagger}$ Laura Santangelo, ${ }^{\S}$ Cristina Di Giacomo, ${ }^{\dagger}$ Viviana Costa,${ }^{\dagger}$ Laura Amicone, ${ }^{\S}$ Giuseppe Ippolito, ${ }^{\dagger}$ Leopoldo Paolo Pucillo, ${ }^{\dagger}$ Tonino Alonzi, ${ }^{\dagger}$ and Marco Tripodi ${ }^{*},{ }^{\dagger}, \S$

${ }^{\dagger}$ L. Spallanzani National Institute for Infectious Diseases, IRCCS, via Portuense 292, 00149, Rome, Italy

${ }^{\S}$ Istituto Pasteur-Fondazione Cenci Bolognetti, Department of Cellular Biotechnologies and Haematology, Sapienza University of Rome, Italy

Supporting Information

ABSTRACT: Hepatic fat export occurs by apolipoprotein B-100-containing lipoprotein production, whereas impaired production leads to liver steatosis. Hepatitis $\mathrm{C}$ virus (HCV) infection is associated to dysregulation of apoB-100 secretion and steatosis; however, the molecular mechanism by which $\mathrm{HCV}$ affects the apoB-100 secretion is not understood. Here, combining quantitative proteomics and computational biology, we propose ferritin heavy chain (Fth) as being the cellular determinant of apoB-100 production inhibition. By means of molecular analyses, we found that HCV nonstructural proteins and NS5A appear to be sufficient for inducing Fth up-regulation. Fth in turn was found to inhibit apoB-100 secretion leading to increased intracellular degradation via proteasome. Notably, intracellular Fth down-regulation by siRNA restores apoB-100 secretion. The inverse correlation between ferritin and plasma apoB100 concentrations was also found in JFH-1 HCV cell culture systems (HCVcc) and HCV-infected patients. Finally, Fth expression was found to be required for robust HCV infection. These observations provide a further molecular explanation for the onset of liver steatosis and allow for hypothesizing on new therapeutic and antiviral strategies.

KEYWORDS: liver steatosis, hypobetalipoproteinemia, HCV infection, iron metabolism

\section{INTRODUCTION}

Hepatitis $\mathrm{C}$ virus ( $\mathrm{HCV}$ )-associated morbidity and mortality are mainly attributable to disease progression toward cirrhosis and, consequently, hepatocellular carcinoma. ${ }^{1}$ In particular, the alterations of lipid metabolism associated with HCV-chronic infection, mainly hypobetalipoproteinemia (HBL) and hepatic steatosis, are considered key risk factors in disease progression. ${ }^{2,3} \mathrm{HBL}$ is a group of metabolic disorders characterized by low levels of circulating apoB-100-containing lipoprotein particles (VLDL and LDL). In particular, reduced production of VLDL determines hepatic fat accumulation, often resulting in steatosis. ${ }^{4} \mathrm{HBL}$ is mainly caused by the impaired secretion of apolipoprotein B-100 (apoB-100), the main constituent of these lipoproteins. ${ }^{5}$ In fact, the VLDL secreted levels are directly correlated to the balance between apoB-100 synthesis and degradation processes.

VLDL synthesis occurs in two different stages: assembly and intracellular maturation. ${ }^{6}$ In the first step, microsomal triglyceride transfer protein (MTP), an endoplasmatic reticulum (ER)-resident enzyme, promotes the addition of small amounts of triglycerides (TGs) to apoB-100, while it translocates across the ER membranes. ${ }^{7,8}$ In the second step,
VLDL precursors are fused with TG-rich droplets to form mature and secretion-competent VLDL particles.

In genetic forms of HBL, mutations of the MTP gene lead to the production of an abnormally short enzyme with reduced or absent activity that generates poorly lipidated apoB-100. ${ }^{9}$ In the absence of sufficient lipids, apoB-100 is unable to form mature VLDL particles and is targeted to both co- and posttranslationally proteasome-dependent degradation. ${ }^{10}$ Similarly, nonsense, frame-shift and splicing mutations of the apoB-100 gene generate truncated forms of the protein that are cotranslationally ubiquitinated and degradated, thus leading to HBL. ${ }^{11}$

While the rationale of several HBL genetic forms has been clarified, the molecular mechanism responsible for the HCVassociated low plasma apoB-100 concentration is yet unknown. Although experimental models (i.e., subgenomic replicon harboring hepatoma cell lines) allowed for pinpointing a causal correlation between expression of the sole HCV nonstructural (NS) proteins and the drastic inhibition of apoB-100 secretion, ${ }^{12}$

Received: November 12, 2011

Published: March 23, 2012 
the basis of the viral-induced inhibition of apoB-100 secretion remains largely unknown.

The present study aimed to address this issue. By combining stable isotope labeling with amino acids in cell culture (SILAC)-based proteomic approach, computational interpretation of data set and molecular analysis we demonstrated that: (i) the expression of HCV nonstructural proteins, and NS5A appears to be sufficient, directly correlates with ferritin heavy chain (Fth) up-regulation; (ii) HCV-induced Fth up-regulation results in inhibition of apoB-100 secretion and in its increased proteasome-associated degradation; (iii) Fth down-regulation abolishes HCV-mediated apoB-100 secretion inhibition. Notably, in a natural infection system (JFH-1 HCVcc), Fth is required to sustain viral infection. Moreover, studies in $\mathrm{HCV}$ infected patients showed that circulating apoB-100 levels were lower in donors with high ferritin values than in those with normal ferritin concentrations.

\section{EXPERIMENTAL SECTION}

\section{Ethics Statement}

The study has been approved by the ethics committee of the INMI "L. Spallanzani" IRCCS, Rome, Italy. This study is included in the INMI "L. Spallanzani" IRCCS research program entitled "Programma di ricerca corrente 2008".

Analysis on human serum has been carried out on clinical residual samples and analyzed anonymously. To protect patient confidentiality, all patient identifiers were removed from the samples to make them completely anonymous.

\section{Reagents}

The chemicals, reagents and suppliers that were used are listed below: $N, N, N^{\prime}, N^{\prime}$-tetrametiletilenediammine (TEMED), Sodium Dodecyl Sulfate (SDS), Tween 20, Albumin Serum Bovine (BSA), Nonidet-p40, Phosphate Buffered Saline (PBS), Dulbecco's modified eagle medium (DMEM), Dulbecco's modified eagle medium nutrient mixture F-12 ham (DMEM F-12), Trypsin-EDTA solution, Fetal Bovine Serum (FBS), L-Glutamine, penicillin/streptomycin, trifluoroacetic acid, interferon-alpha (IFN- $\alpha$ ), Ponceau S solution, $\alpha$-cyano-4-hydroxycinnamic acid (CHCA), paraformaldehyde, Triton X-100, donkey serum, oligonucleotides siCTR/siFTH-1, sodium chloride $(\mathrm{NaCl}), \mathrm{EDTA}$, protease inhibitor cocktail (PIC), dl-dithiothreitol (DTT), iodoacetamide (IAA), MG-132, $N$-acetyl cysteine (NAC) and ammonium bicarbonate $\left(\mathrm{NH}_{4} \mathrm{HCO}_{3}\right)$ were purchased from Sigma-Aldrich (St. Louis, MO). Acrylamide, Tris-HCl Buffer, Tris-glycine-SDS buffer, Bradford Bio-Rad Protein Assay, Sypro Ruby gel stain, Precision Plus Protein Standard were purchased from BioRad Laboratories (Hercules, CA). Blasticidin and sucrose were purchased from ICN Biomedical (Costa Mesa, CA). NuPAGE gel electrophoresis reagents, lipofectamine RNAiMAX reagent, SILAC media DMEM-Flex, TRIzol reagent and 5-6-chloromethyl-2', 7 'dichlorodihydrofluorescein diacetate (CM- $\left.\mathrm{H}_{2} \mathrm{DCFDA}\right)$ were obtained from Invitrogen (Carlsbad, CA). Nytrocellulose membranes (Protran BA85) were obtained from WhatmanSchleicher \& Schuel (Dassel, Germany). ECL Plus Western Blotting Detection System was purchased from GE Healthcare (Chalfont St Giles, U.K.). Methanol and acetonitrile (ACN) were purchased from J.T. Baker (Phillipsburg, NJ). Vivaspin $15 \mathrm{R}$ ultracentrifuge columns were purchased from Sartorius Stedim Biotech (Germany) and microcolumns ZipTip ( $\mu$-C18) were purchased from Millipore Corporation (Ballerica, USA). Anti-Calnexin, MTP, NS5A, and Albumin antibodies were purchased from Santa Cruz Biotechnology (Santa Cruz, USA); anti-Calreticulin and ERP57 antibodies were obtained from Stressgen Biotechnologies (San Diego, CA). Anti-ApolipoproteinB-100 antibody was purchased from BD Biosciences (San Jose, CA); anti-Ferritin heavy chain antibody was obtained from AbCam (Cambridge, U.K.). Anti-GAPDH antibody was purchased from Calbiochem Merck (Gibbstown, NJ) and antiGolgin97 antibody was obtained from Invitrogen (Carlsbad, CA). Anti-Core antibody was purchased from Anogen (Mississauga, Canada). Secondary antibodies were purchased from Jackson Immunoresearch Laboratories (Baltimore, MD).

\section{Cell Culture}

All cells were maintained in a monolayer at $37{ }^{\circ} \mathrm{C}$ in $5 \% \mathrm{CO}_{2}$. For the proteasome inhibition assay, RepBlast and Huh7 cells were incubated in DMEM F-12 for $3 \mathrm{~h}$ in the presence of MG$13210 \mu \mathrm{M}$. Cells were then lysed on ice with RIPA lysis buffer (50 mM Tris $\mathrm{HCl} \mathrm{pH} 8.0,150 \mathrm{mM} \mathrm{NaCl}, 1 \mathrm{mM}$ EDTA, $5 \mathrm{mM}$ EGTA, 1\% Nonidet NP-40, 0.5\% sodium deoxycholate, $0.1 \%$ sodium dodecyl sulfate) containing freshly added protease inhibitors and $1 \mathrm{mM}$ PMSF. Cellular medium was collected, centrifuged $\left(17000 \times \mathrm{g}, 4{ }^{\circ} \mathrm{C}, 10 \mathrm{~min}\right)$ to remove cells and debris, and secreted proteins were concentrated into ultrafiltration columns. Samples were analyzed by Bradford assay to determine the protein concentration, then dissolved in SDSloading buffer and analyzed by Western blotting. For IFN $\alpha$ treatment, $2 \times 10^{5}$ RepBlast and Huh7 plated in 6 -cm dishes were incubated in DMEM and then treated with IFN $\alpha 1000 \mathrm{U} / \mathrm{mL}$. The media was changed every $24 \mathrm{~h}$ for 4 days.

For HCVcc production, Huh7.5.1 cells were infected with JFH-1-derived virus $(\mathrm{MOI}=0.1) 2$ days before plating. After 3 days of cultivation, the cells were lysed and culture media were collected as previously described.

\section{Membrane Floatation Assay}

Gradients were formed by equal-volume $(1,5 \mathrm{~mL})$ steps of 20 , $30,40,50$, and $60 \%$ sucrose solutions in TNE buffer $(10 \mathrm{mM}$ Tris-HCl, pH 8, $150 \mathrm{mM} \mathrm{NaCl}, 2$ mM EDTA). Eight-hundred micrograms of sample $(1.5 \mathrm{~mL})$ were overlaid on the gradient, and equilibrium was reached by ultracentrifugation for $16 \mathrm{~h}$ at $33000 \mathrm{rpm}(135000 \times \mathrm{g})$ in an SW41Ti rotor at $4{ }^{\circ} \mathrm{C}$ in a Beckman Coulter optima L-90K preparative ultracentrifuge. Gradient fractions $(13 \times 600 \mu \mathrm{L})$ were collected from the top and the density of the fractions was determined by measuring the mass of $100-\mu \mathrm{L}$ aliquots of each sample.

\section{SILAC Labeling of Human Cultured Hepatocytes}

RepBlast and Huh7 cells were grown respectively in SILAC "light" (L-arginine and L-lysine) and "heavy" ( $\mathrm{L}^{-13} \mathrm{C}_{6}{ }^{15} \mathrm{~N}_{4}$ arginine and $\mathrm{L}^{-}{ }^{13} \mathrm{C}_{6}$ - lysine) conditions for 8 passages before the experiment. This period lasted about 3 weeks. Cells were lysed in hypotonic buffer $(0.05 \%$ Nonidet P-40, $10 \mathrm{mM}$ HEPES/KOH pH 7.9, $1.5 \mathrm{mM} \mathrm{MgCl} 2,150 \mathrm{mM} \mathrm{NaCl}, 10 \mathrm{mM}$ $\mathrm{KCl}, 1 \mathrm{mM}$ sodium orthavanadate, $5 \mathrm{mM} \mathrm{NaF}, 0.5 \mathrm{mM}$ DTT and protease inhibitors) at four degrees with rotation for 30 min. The lysates were cold centrifuged with $17000 \times g$ for 15 min to pellet nuclei and cellular debris. Supernatants (PNCE) were collected, and the Bradford method was used to determine the protein concentrations. Equal amounts (400 $\mu \mathrm{g} / 750 \mu \mathrm{L})$ of the proteins from the Huh7 and RepBlast samples were mixed and analyzed by membrane floatation and nanoLC-MALDI. Two independent biological and technical replicates were performed. 


\section{Western Blotting Assay}

Proteins were separated on Tris-Acetate 3-8\% and Bis-Tris 4$12 \%$ gradient polyacrylamide gels (Invitrogen) and transferred to a nitrocellulose membrane. Membranes were then blocked with $0.5 \%$ Tween-PBS containing $5 \%$ nonfat dried milk and incubated overnight with the primary antibodies followed by incubation with HRP-conjugated species-specific secondary antibodies and enhanced chemiluminescence reaction using ECL Plus Western Blotting Detection System.

Protein Digestion, Peptide Purification and nanoLC Analysis

Membrane floatation-derived fractions 7, 8, and 9 obtained from the SILAC experiment were pooled and $100 \mu \mathrm{g}$ of proteins were separated on 4-12\% gradient gels (Invitrogen), stained by Sypro Ruby staining and visualized using a Typhoon 9200 laser scanner (GE Healthcare). Fourteen sections of the gel lane were cut. Protein-containing gel pieces were washed with $100 \mu \mathrm{L}$ of $0.1 \mathrm{M}$ ammonium bicarbonate ( $5 \mathrm{~min}$ at $\mathrm{RT}$ ). Then, $100 \mu \mathrm{L}$ of $100 \%$ acetonitrile (ACN) was added to each tube and incubated for $5 \mathrm{~min}$ at RT. The liquid was discarded, the washing step repeated once more, and the gel plugs were shrunk by adding ACN. The dried gel pieces were reconstituted with $100 \mu \mathrm{L}$ of $10 \mathrm{mM}$ DTT/0.1 M ammonium bicarbonate and incubated for $40 \mathrm{~min}$ at $56{ }^{\circ} \mathrm{C}$ for cysteine reduction. The excess liquid was then discarded and cysteines were alkylated with $100 \mu \mathrm{L}$ of $55 \mathrm{mM}$ IAA/0.1 M ammonium bicarbonate (20 min at RT, in the dark). The liquid was discarded, the washing step was repeated once more, and the gel plugs were shrunk by adding ACN. The dried gel pieces were reconstituted with 12.5 $\mathrm{ng} / \mu \mathrm{L}$ trypsin in $50 \mathrm{mM}$ ammonium bicarbonate and digested overnight at $37{ }^{\circ} \mathrm{C}$. The supernatant from the digestion was saved in a fresh tube and $100 \mu \mathrm{L}$ of $1 \%$ TFA/30\% ACN were added on the gel pieces for an additional extraction of peptides. The extracted solution and digested mixture were then combined and vacuum centrifuged for organic component evaporation. Peptides were resuspended with $40 \mu \mathrm{L}$ of $2.5 \%$ ACN/0.1\% TFA, desalted and filtered through a C18 microcolumn ZipTip, and eluted from the C18 bed using $10 \mu \mathrm{L}$ of $80 \%$ ACN/0.1\% TFA. The organic component was once again removed by evaporation in a vacuum centrifuge and peptides were resuspended in a suitable nanoLC injection volume (typically $3-10 \mu \mathrm{L}$ ) of $2.5 \% \mathrm{ACN} / 0.1 \%$ TFA. An UltiMate 3000 nano-LC system (Dionex, Sunnyvale, CA) equipped with an integrated nanoflow manager and microvacuum degasser was used for peptide separation. The peptides were loaded onto a $75 \mu \mathrm{m}$ I.D. NanoSeries C18 column (Dionex, P/N 160321) for multistep gradient elution (eluent $\mathrm{A}$ $0.05 \%$ TFA; eluent B $0.04 \%$ TFA in $80 \%$ ACN) from 5 to $20 \%$ eluent $B$ within $10 \mathrm{~min}$, from 20 to $50 \%$ eluent $B$ within 45 min and for further 5 min from 50 to $90 \%$ eluent B with a constant flow of $0.3 \mu \mathrm{L} / \mathrm{min}$. After $5 \mathrm{~min}$, the eluted sample fractions were continuously diluted with $0.5 \mu \mathrm{L} / \mathrm{min}$ a-cyano4-hydroxycinnamic acid (CHCA) and spotted onto a MALDI target using a Probot (LC-Packings/Dionex) with an interval of $20 \mathrm{~s}$ resulting in 144 fractions for each gel slice.

\section{Mass Spectrometry Analysis}

MALDI-TOF-MS spectra were acquired using a 4800 Plus MALDI TOF/TOF Analyzer (AB Sciex, Foster City, CA). The spectra were acquired in the positive reflector mode by 20 subspectral accumulations (each consisting of 50 laser shots) in an 800-4000 mass range, focus mass $2100 \mathrm{Da}$, using a $355 \mathrm{~nm}$ $\mathrm{Nb}$ :YAG laser with a $20 \mathrm{kV}$ acceleration voltage. Peak labeling was automatically done by 4000 Series Explorer software Version 3.0 (AB Sciex) without any kind of smoothing of peaks or baseline, considering only peaks that exceeded a signal-tonoise ratio of 10 (local noise window $200 \mathrm{~m} / z$ ) and a half maximal width of 2.9 bins. Calibration was performed using default calibration originated by five standard spots (ABI4700 Calibration Mixture). Only MS/MS spectra of preselected peaks (out of peak pairs with a mass difference of 6.02, 10.01, $12.04,16.03$, and $20.02 \mathrm{Da}$ ) were integrated over 1000 laser shots in the $1 \mathrm{kV}$ positive ion mode with the metastable suppressor turned on. Air at the medium gas pressure setting $\left(1.25 \times 10^{-6}\right.$ Torr $)$ was used as the collision gas in the CID off mode. After smoothing and baseline subtractions, spectra were generated automatically by 4000 Series Explorer software. MS and MS/MS spectra were processed by ProteinPilot Software 2.0.1 (AB SCIEX) which acts as an interface between the Oracle database containing raw spectra and a local copy of the MASCOT search engine (Version 2.1, Matrix Science, Ltd.). The Paragon algorithm was used with SILAC (Lys+6, Arg+10) selected as the Sample Type, iodacetamide as cysteine alkylation, with the search option "biological modifications" checked, and trypsin as the selected enzyme. MS/MS protein identification was performed against the Swiss-Prot database (number of protein sequences: 254757; released on 20070123) without taxon restriction using a confidence threshold of $95 \%$ (Proteinpilot Unused score $\geq 1.31$ ). The monoisotopic precursor ion tolerance was set to $0.12 \mathrm{Da}$ and the MS/MS ion tolerance to $0.3 \mathrm{Da}$. The minimum required peptide length was set to 6 amino acids; two peptides were required for protein identification.

For quantitation, the Heavy/Light average ratio for a protein was calculated by ProteinPilot Software with automatic bias correction. Quantitation was based on a two-dimensional centroid of the isotope clusters within each SILAC pair. Ratios of the corresponding isotope forms in the SILAC pair were calculated, and lines fitting these intensity ratios gave the slope as the desired peptide ratio. To represent the ratio of a peptide being quantified several times, the median value was chosen. To minimize the effect of outliers, protein ratios were calculated as the median of all SILAC pair ratios that belonged to peptides contained in this protein. The percentage of quantitation variability was defined as the standard deviation of the natural logarithm of all ratios used for obtaining the protein ratio multiplied by a constant factor of 100 .

Only relative Heavy/Light (or Light/Heavy) ratios exceeding factor 1.5 were considered. Relative protein and peptides information and raw mass spectra were exported to an Excel file (Supplemental data 2, Supporting Information).

\section{Data Analysis}

Data were analyzed using Ingenuity Pathways Analysis. A data set containing gene identifiers and corresponding expression values from proteomics analysis was uploaded into the application. Each identifier was mapped to its corresponding object in the Ingenuity Knowledge Base. A Heavy/Light ratio cutoff point of 1.5 was set to identify molecules whose expression was significantly differentially regulated. These molecules, called Network Eligible molecules, were overlaid onto a global molecular network developed from information contained in the Ingenuity Knowledge Base. Networks of Network Eligible Molecules were then algorithmically generated based on their connectivity. The Functional Analysis of a network identified the biological functions and/or diseases 
that were most significant to the molecules in the network. The network molecules associated with biological functions and/or diseases in the Ingenuity Knowledge Base were considered for the analysis. Right-tailed Fisher's exact test was used to calculate a $p$-value determining the probability that each biological function and/or disease assigned to that network is due to chance alone. A network is a graphical representation of the molecular relationships between molecules. Molecules are represented as nodes, and the biological relationship between two nodes is represented as an edge (line). All edges are supported by at least 1 reference from the literature, a textbook, or from canonical information stored in the Ingenuity Pathways Knowledge Base. Human, mouse, and rat orthologs of a gene are stored as separate objects in the Ingenuity Pathways Knowledge Base, but are represented as a single node in the network. The intensity of the node color indicates the degree of up- (green) or down- (red) regulation. Nodes are displayed using various shapes that represent the functional class of the gene product. Edges are displayed with various labels that describe the nature of the relationship between the nodes.

\section{ApoB-100 Secretion in Human Cultured Hepatocytes}

Protein secretion from cultured hepatocytes was performed in a serum-free medium for $16 \mathrm{~h}$. The medium was then removed, centrifuged $\left(17000 \times \mathrm{g}, 4{ }^{\circ} \mathrm{C}, 10 \mathrm{~min}\right)$ to remove cells and debris, and secreted proteins were concentrated into ultrafiltration columns. Samples were then dissolved in an SDSloading buffer and analyzed for apoB-100 by Western blotting.

ApoB-100 and Ferritin Levels in Human Plasma Samples

Plasma ferritin and apoB-100 concentrations were assessed in a centralized laboratory using automated laboratory methods.

\section{Production of Recombinant Retroviruses}

To produce recombinant replication-incompetent retroviruses, 293GP packaging cells were transiently transfected according to standard procedures with a pLPCX retroviral construct (BD Biosciences, San Jose, CA) encoding HCV NS5a (genotype 1b) or the control beta-Galactosidase protein, in the presence of a plasmid-encoding VSV envelope protein. Culture supernatants were collected at $48 \mathrm{~h}$ after transfection, filtered at $0.45 \mathrm{~mm}$, concentrated by ultracentrifugation and frozen.

RNA Extraction, Reverse Transcription and Quantitative Real-time PCR

Total RNA was extracted by TRIzol and $1 \mu \mathrm{g}$ was reverse transcribed with MMLV-reverse-transcriptase (Promega, Madison, WI). Quantitative real time PCR was performed on 1:10 cDNA dilution using BioRad Miniopticon with KAPA SYBR Green FAST qPCR mix (KAPABIOSYSTEMS, Woburn, MA). Cycling parameters were: $95{ }^{\circ} \mathrm{C}$ for $3 \mathrm{~min}$, followed by 40 cycles of $95{ }^{\circ} \mathrm{C}$ for 10 s, $59{ }^{\circ} \mathrm{C}$ for 30 s, $72{ }^{\circ} \mathrm{C}$ for 10 s. The relative amount of Fth mRNA was obtained by $2^{-\Delta \Delta C t}$ method and normalized to human L34 gene expression. The following primers were designed with PerIPrimer software: L34 sense 5'-GTCCCGAACCCCTGGTAATAG-3'; L34 antisense 5'-GGCCCTGCTGACATGTTTCTT-3'; Fth sense 5'-TACTGGAACTGCACAAACTG-3'; Fth antisense 5'-GCTTTCATTATCACTGTCTCCC-3'; Ftl sense 5'-GCTACGAGCGTCTCCTGAAG-3'; and Ftl antisense 5'-ATGGCGTCTGGGGTTTTAC-3'. Data represents the mean of triplicate experiments and statistical analysis was based on Student's $t$ test. HCV RNA copy number quantification on JFH-1-infected cells were determined as described by Mancone et al. ${ }^{14}$
siRNA Transfections and NS5a Infections

Ds-siRNA Fth-specific (sense: 5'-GAAUCAGUCACUACUGGAACdTdT-3'; antisense: 5'-GUUCCAGUAGUGACUGAUUCdTdT-3') and ds-siRNA Ftl-specific (sense: 5'-GUCAGAAUUAUUCCACCGAC-3'; antisense 5'-GUCGGUGGAAUAAUUCUGAC-3'). The Mission Universal Negative Control siRNA was purchased from Sigma-Aldrich (St. Louis, MO). For ds-siRNA transfection, $4.5 \times 10^{5}$ RepBlast cells were plated in $60 \mathrm{~mm}$ dishes and transfected with 400 pmoles of Fth-specific or negative control ds-siRNA by Lipofectamine RNAiMAX (Invitrogen, Carlsbad, CA) following the manufacturer's instructions. The day after transfection, cells were washed twice with PBS and cultured in DMEM F12 medium without serum for $16 \mathrm{~h}$. Cell-conditioned medium was then collected at $48 \mathrm{~h}$ after ds-siRNA transfection, and concentrated as described previously. Cells were lysed on ice with RIPA lysis buffer ( $50 \mathrm{mM}$ Tris $\mathrm{HCl} \mathrm{pH} 8.0,150 \mathrm{mM} \mathrm{NaCl}, 1 \mathrm{mM}$ EDTA, $5 \mathrm{mM}$ EGTA, 1\% Nonidet NP-40, 0.5\% sodium deoxycholate, $0.1 \%$ sodium dodecyl sulfate) containing freshly added protease inhibitors and $1 \mathrm{mM}$ PMSF. Lysates were clarified by centrifugation at $13000 \mathrm{rpm}$ for $15 \mathrm{~min}$ at $4{ }^{\circ} \mathrm{C}$ and protein concentrations were determined by Bradford assay using BSA as a standard.

In experiments where Fth knock-down was followed by NS5a retroviral infection, $1.5 \times 10^{5}$ Huh-7 cells were plated in $35 \mathrm{~mm}$ dishes and transfected with 200 pmoles of Fth-specific or negative control ds-siRNA. Cell culture medium was changed six hours after transfection and an overnight infection was performed with NS5a and the control beta-Galactosidase retroviral supernatants, respectively, in the presence of $4 \mu \mathrm{g} / \mathrm{mL}$ Polybrene. The day after transfection, cells were washed twice with PBS and cultured in DMEM F12 without serum for $3 \mathrm{~h}$. Cellconditioned medium and total lysates were collected and analyzed at $48 \mathrm{~h}$ after transfection.

Analysis of ROS concentration was performed on Huh7 infected with a retroviral vector expressing NS5A and treated with Fth-specific or negative control ds-siRNA by 5-6-chloromethyl$2^{\prime}, 7^{\prime}$-dichlorodihydrofluorescein diacetate (CM- $\left.\mathrm{H}_{2} \mathrm{DCFDA}\right)$ assay according to the manufacturer's instructions. Cells were incubated with $\mathrm{CM}-\mathrm{H}_{2}$ DCFDA $(10 \mu \mathrm{M})$ for $20 \mathrm{~min}$ at $37^{\circ} \mathrm{C}$ in the dark, washed in cold PBS and subjected to cytofluorimetric analysis by FACSCalibur (BD Biosciences, CA).

\section{Immunofluorescent Microscopy}

For immunofluorescence analysis, cells were fixed with $4 \%$ paraformaldehyde, permeabilized with $0.2 \%$ Triton, blocked with $10 \%$ donkey serum and then incubated with an antiCORE antibody (Anogen) for $1 \mathrm{~h}$. After being washed, cells were incubated with CyTM2-conjugated AffiniPure $\mathrm{F}\left(\mathrm{ab}^{\prime}\right)_{2}$ fragment donkey antimouse IgG secondary antibody (ImmunoResearch Laboratories, Inc.) for $30 \mathrm{~min}$. Cells were counterstained with DAPI to label nuclei.

\section{RESULTS}

HCV Nonstructural Proteins Modulate the Composition of apoB-100 Containing Subcellular Fractions

The development of selectable subgenomic HCV RNA replicons has led to important advances in the study of the relationship between HCV and the host lipoprotein metabolism. ${ }^{12,14}$ In this study, we made use of RepBlast, a HCV subgenomic (genotype $1 \mathrm{~b}$ ) replicon cell line derived from a mixed population of Huh7 cells that allow for minimizing clone-specific effects. ${ }^{14}$ First, we assessed if HCV NS expression 

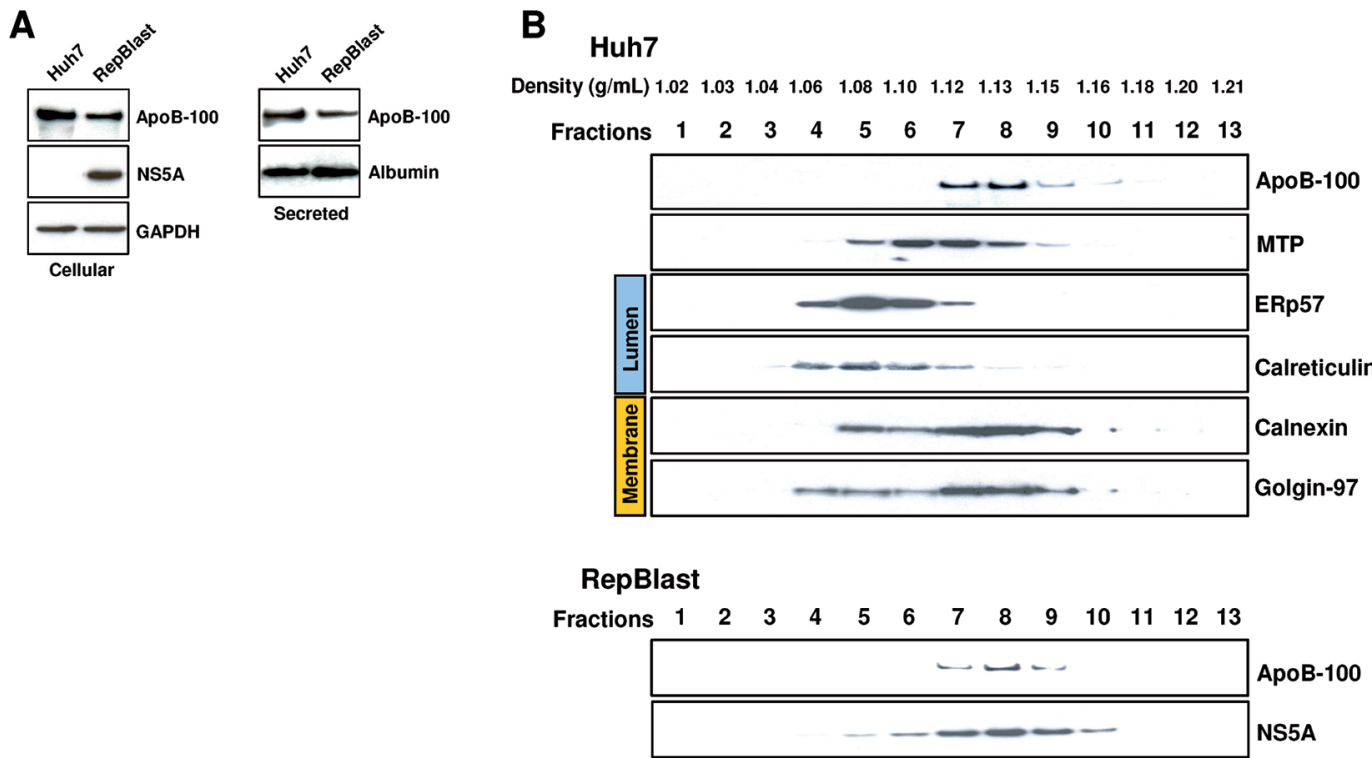

Figure 1. Characterization of apoB-100-containing vesicles. (A) Cellular and secreted apoB-100 Western blot analysis in the indicated cell lines. NS5A was used as control for HCV-replicon expression; GAPDH and albumin for protein loading control. (B) Western blot analysis for apoB-100 intracellular distribution. Equal volumes of membrane floatation-derived vesicles isolated from both Huh7 and RepBlast cells were analyzed for membrane (MTP, Calnexin and Golgin-97) and lumenal (MTP, Erp57 and Calreticulin) markers. The viral protein NS5A was used as control for HCV-replicon intracellular distribution.

affects the apoB-100 production of RepBlast cells. As expected, the intracellular and secreted apoB-100 levels were found to be decreased when compared with counterpart replicon-free cells (Huh7) (Figure 1A).

To identify possible cellular effectors responsible for the HCV-mediated inhibition of apoB-100 production, we endeavored to make an unbiased characterization of apoB-100 containing subcellular fractions. This required investigating the multistep process spanning from apoB-100 synthesis to secretion. RepBlast and Huh7 cells subcellular fractions were initially isolated from postnuclear cell extracts (PNCE) by membrane floatation. The obtained fractions were then analyzed for the distribution of apoB-100 by Western blotting (Figure 1B). ApoB-100 was detected in density fractions ranging from 1.12 to $1.16 \mathrm{~g} / \mathrm{mL}$ (i.e.: fractions $7-9$ ), as expected from the predicted membrane-associated biogenesis of VLDL (Figure 1B and Supplemental data 1, Supporting Information).

Currently, several strategies for differential proteomics that involve stable isotopes or label-free comparisons are available, each having specific strengths and limitations. To characterize differences in apoB-100 containing fractions between RepBlast and Huh7 cells, we chose the SILAC proteomic approach that allows for mixing samples before floatation assay, thus circumventing sample differences due to the generation of distinct density gradients (Figure 2). To this end, we metabolically labeled Huh7 cells with ${ }^{13} \mathrm{C}_{6}{ }^{15} \mathrm{~N}_{4}$-arginine and ${ }^{13} \mathrm{C}_{6}$-lysine (Heavy) while RepBlast cells were grown in light medium. PNCE were then isolated separately and equal amounts of protein from each cell line were mixed. After membrane flotation, apoB-100 containing fractions (7-9) were pooled, separated by SDS-PAGE and the gel lane was cut into 14 sections ranging from 15 to $250 \mathrm{kDa}$. Proteins in each gel section were digested and submitted to nanoLC-MALDITOF/TOF analysis. We considered proteins differentially expressed when the SILAC ratio was Heavy/Light or Light/Heavy $\geq 1.5$ and

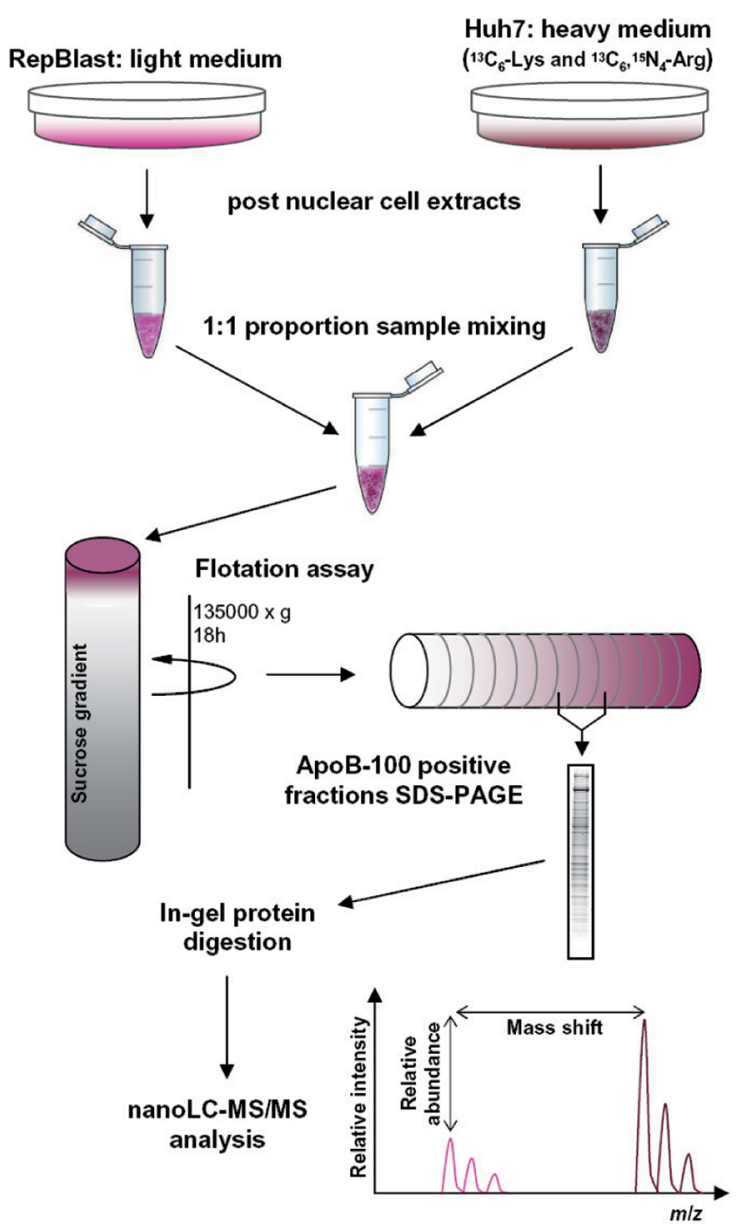

Figure 2. Schematic representation of SILAC-based proteomic approach on membrane floatation-derived apoB-100 positive fractions.

at least two peptides were found fragmented by MS/MS in two independent biological replicates. 
Table 1. HCV-Induced Protein Modulations in apoB-100-Containing Fractions (7-9) Identified by nanoLiquid Chromatography-Mass Spectrometry/Mass Spectrometry

\begin{tabular}{|c|c|c|c|c|c|c|}
\hline acc. number ${ }^{a}$ & gene name ${ }^{a}$ & protein name & SILAC average ratio $\pm \mathrm{SD}^{b}$ & protein score ${ }^{c}$ & seq. coverage $\%$ & number of peptides \\
\hline \multicolumn{7}{|c|}{ HCV-replicon up-regulated proteins (Light/Heavy) } \\
\hline P01023 & $\mathrm{A} 2 \mathrm{M}$ & Alpha-2-macroglobulin & $6.62 \pm 4.79$ & 5.15 & 9.2 & 13 \\
\hline P38646 & HSPA9 & Stress- 70 protein, mitochondrial & $4.79 \pm 4.11$ & 13.34 & 26.2 & 18 \\
\hline Q9BSE5 & AGMAT & Agmatinase, mitochondrial & $4.66 \pm 3.51$ & 4.00 & 8.5 & 3 \\
\hline P00505 & GOT2 & Aspartate aminotransferase, mitochondrial & $4.00 \pm 3.18$ & 8.32 & 17.9 & 8 \\
\hline Q9BPW8 & NIPSNAP1 & Protein NipSnap1 homologue 1 & $3.62 \pm 2.73$ & 6.11 & 23.9 & 6 \\
\hline P30084 & ECHS1 & Enoyl-CoA hydratase, mitochondrial & $3.45 \pm 2.20$ & 7.64 & 56.6 & 16 \\
\hline Q15738 & NSDHL & Sterol-4-alpha-carboxylate 3-dehydrogenase & $2.63 \pm 1.51$ & 4.10 & 11.0 & 3 \\
\hline P58107 & EPPK1 & Epiplakin & $2.42 \pm 1.29$ & 22.85 & 40.3 & 71 \\
\hline P02792 & FTL & Ferritin light chain & $2.32 \pm 1.29$ & 11.56 & 68.6 & 14 \\
\hline P27824 & CANX & Calnexin & $2.21 \pm 0.69$ & 5.32 & 14.2 & 9 \\
\hline P05091 & ALDH2 & Aldehyde dehydrogenase, mitochondrial & $2.11 \pm 0.09$ & 8.00 & 22.2 & 8 \\
\hline P02794 & FTH1 & Ferritin heavy chain & $2.02 \pm 0.68$ & 11.49 & 57.4 & 11 \\
\hline Q16881 & TXNRD1 & Thioredoxin reductase 1 , cytoplasmic & $1.63 \pm 0.42$ & 9.60 & 44.9 & 19 \\
\hline Q04828 & AKR1C1 & Aldo-keto reductase family 1 member $\mathrm{C} 1$ & $1.56 \pm 0.00$ & 16.89 & 63.2 & 15 \\
\hline P16279 & GLB1 & Beta-galactosidase & $1.53 \pm 0.20$ & 5.22 & 20.9 & 12 \\
\hline $\mathrm{O} 15254$ & ACOX3 & Peroxisomal acyl-coenzyme A oxidase 3 & $1.50 \pm 0.01$ & 4.00 & 17.4 & 10 \\
\hline \multicolumn{7}{|c|}{ HCV-replicon down-regulated proteins (Heavy/Light) } \\
\hline P12277 & $\mathrm{CKB}$ & Creatine kinase B-type & $1.90 \pm 0.39$ & 26.27 & 66.4 & 19 \\
\hline Q01581 & HMGCS1 & Hydroxymethylglutaryl-CoA synthase & $1.86 \pm 0.23$ & 14.01 & 32.3 & 14 \\
\hline Q16555 & DPYSL2 & Dihydropyrimidinase-related protein 2 & $1.60 \pm 0.10$ & 17.35 & 46.0 & 23 \\
\hline Q9Y617 & PSAT1 & Phosphoserine aminotransferase & $1.57 \pm 0.27$ & 13.98 & 29.7 & 9 \\
\hline P04114 & APOB & Apolipoprotein B-100 & $1.52 \pm 0.02$ & 16.11 & 19.2 & 83 \\
\hline P68363 & TUBA1B & Tubulin alpha-1B chain & $1.52 \pm 0.27$ & 34.05 & 55.4 & 19 \\
\hline Q9BQE3 & TUBA1C & Tubulin alpha-1C chain & $1.51 \pm 0.33$ & 5.35 & 24.5 & 8 \\
\hline
\end{tabular}

${ }^{a}$ According to the UniProtKB/Swiss-Prot entry. ${ }^{b}$ Average protein ratios (Heavy/Light and Light/Heavy) and standard deviations (SD) were determined by the mean of two independent biological analyses. For details, see Experimental Section. ${ }^{c}$ ProteinPilot Unused ProtScore (Conf) $>1.30$ $(95.0 \%)$.

By these criteria, we found a total of 23 proteins $(\sim 6 \%$ of the total proteins identified) (Table 1$)$, where 16 were overabundant and 7 were at lower levels in the HCV-replicon harboring cells than in the control cells. Among them, the following 14 were already known to be involved in biological processes perturbed by $\mathrm{HCV}$ infection: ACOX3, NSDHL, APOB, ECHS1 (lipid metabolism), TUBA1B, TUBA1C, DPYSL2, EPPK1 (cytoskeleton remodeling), PSAT1, CANX, GOT2, HSPA9 (protein synthesis and folding), FTL and FTH1 (iron metabolism). ${ }^{15}$

Interaction Network Generated by Proteomic Data Highlights Involvement of Ferritin Heavy Chain in HCV-induced Dysregulation of apoB-100 Secretion

To infer up-regulated proteins in terms of interaction networks or predominant canonical pathways, the differentially regulated proteins identified in this analysis were further investigated in silico by Ingenuity Pathway Analysis (IPA) (Ingenuity Systems, Mountain View, CA; http://www.ingenuity.com). This allowed for generating two networks with associated IPA scores of 45 and 9, respectively (Supporting Information Table 1). We concentrated on data suggesting possible functional significance in the framework of apoB-100-containing lipoprotein production. In particular, network 1 (IPA score 45) highlights that apoB-100 is capable of three physical interactions with proteins found up-regulated in the RepBlast subcellular fractions: calnexin, ferritin light chain (Ftl) and ferritin heavy chain (Fth) (Figure 3A). ${ }^{16,17}$ In particular, the interaction between apoB-100 and Fth (arrow in figure) is based on literature data showing that the induction of Fth expression results in severe inhibition of apoB-100 secretion from hepatoma cells. ${ }^{18}$ Therefore, the combination of our proteomic data with the literature proposes $\mathrm{Fth}$ as the cellular determinant for $\mathrm{HCV}$-induced inhibition of apoB100 production (Figure $3 \mathrm{~B}$ ). We therefore exploited this hypothesis.

HCV-induced Fth Up-regulation Blocks apoB-100 Secretion and Determines its Degradation via Proteasome

First, we confirmed that HCV replicon induces Fth upregulation by Western blot analysis. Fth was found to be upregulated in apoB-100 containing fractions derived from RepBlast cells (Figure 4A). Next, to challenge the causal correlation between viral proteins and cellular Fth upregulation, we induced replicon clearance by prolonged treatment with interferon alpha $(\mathrm{IFN} \alpha)$. As shown in Figure 4B, IFN $\alpha$-treated RepBlast cells showed cellular Fth and apoB100 levels comparable with the control cells. Evidence of the direct influence of Fth expression on the HCV-induced dysregulation of apoB-100 production was achieved by Fthspecific siRNA. As shown in Figure 4C, we found that the Fth down-regulation induced increased intracellular and secreted amounts of apoB-100, in spite of the HCV nonstructural protein expression. Notably, the same analysis carried out on ferritin light chain-silenced RepBlast cells did not highlight effects on apoB-100 (Figure 4D), thus indicating that Fth plays a specific role in apoB-100 production in these hepatoma cell lines.

We then investigated the mechanism that leads to the Fthmediated inhibition of apoB-100 secretion. As mentioned, control of apoB-100 biogenesis occurs by both co- and posttranslational mechanisms. It has been previously demonstrated 


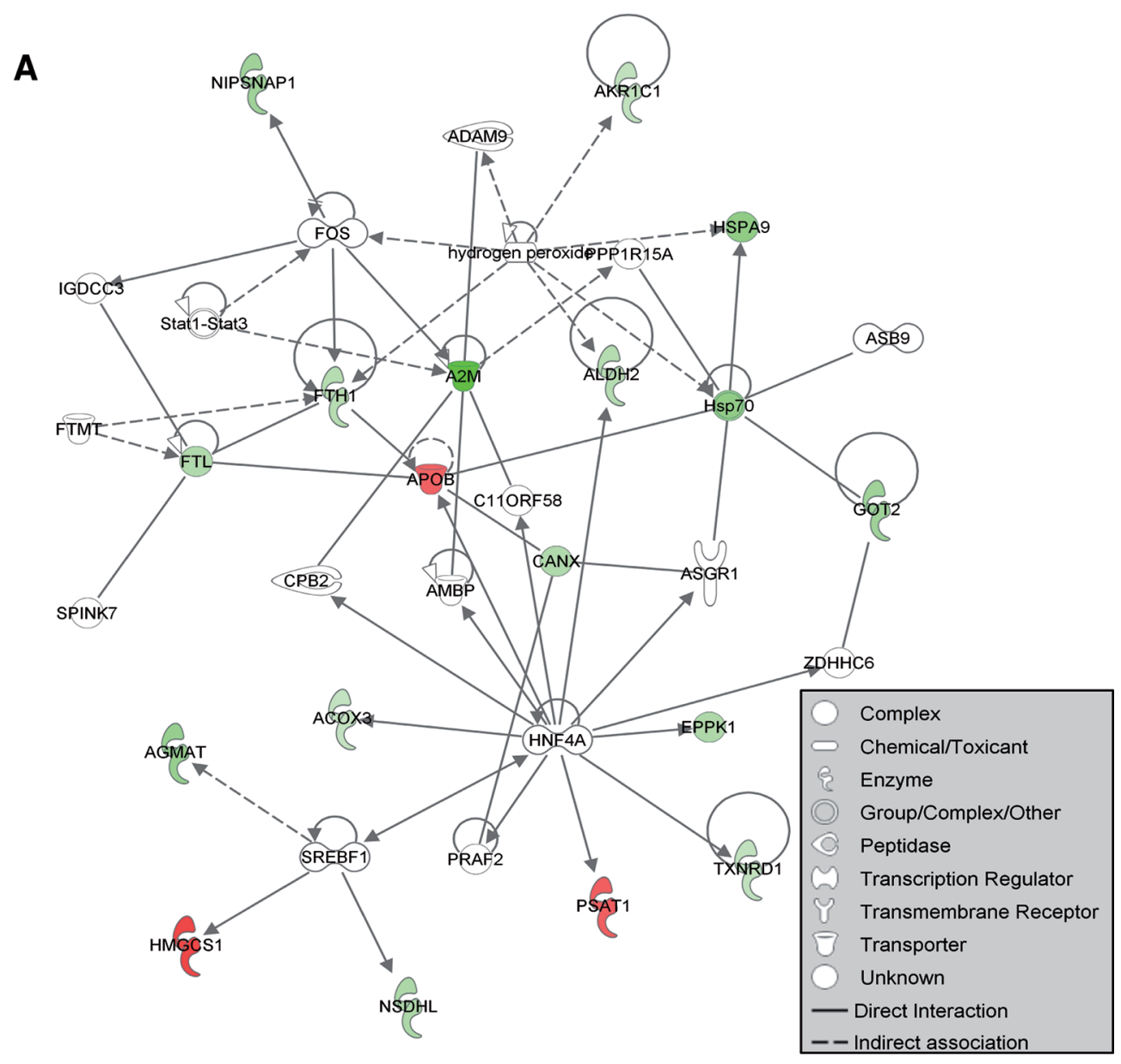

B

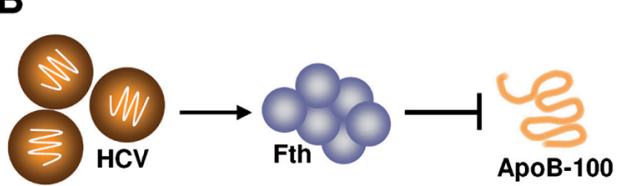

Figure 3. Protein network generation on the apoB-100 structural and functional interactions. (A) SILAC data obtained by comparing the apoB-100 positive fractions of Huh7 and RepBlast cells were analyzed by IPA. HCV-induced protein modulations are indicated in green (up-regulated) and red (down-regulated); arrows denote functional relationships. Symbols are indicated in the legend. (B) Schematic representation of Fth as the cellular determinant of the HCV-induced apoB-100 production inhibition.

that Fth inhibits apoB-100 ER export leading to its increased proteasome-associated degradation. ${ }^{18}$ Therefore, we tested if the $\mathrm{HCV}$-induced Fth up-regulation affects apoB-100 production with the same post-transcriptional mechanism. To this end, we treated Huh7 and RepBlast cell lines with MG-132, a specific inhibitor of proteasome. The intracellular and secreted amounts of apoB-100 were analyzed by Western blotting. When treated with MG-132, RepBlast cells show similar amounts of apoB-100 as control Huh7, while its secretion is inhibited (Figure 4E), thus indicating that apoB-100 increased proteasome degradation is a secondary event that follows inhibition of secretion.

Taken together, these results demonstrate that HCV inhibits apoB-100 production by Fth-mediated post-translational block in secretion.

HCV Nonstructural Protein Expression Induces Fth Up-regulation Independent of Iron-induced and Oxidative Stress-based Mechanisms

Next, we aimed to gain insight on the molecular mechanism by which HCV NS proteins induce Fth expression. It is well- known that Fth synthesis is regulated at a transcriptional level in response to oxidative stress and post-transcriptionally by cytosolic iron. ${ }^{19,20}$ Using RT-qPCR analysis, we found that RepBlast cells expressed statistically significantly higher levels of Fth mRNA in respect to Huh7 (Figure 5A). Notably, the clearance of $\mathrm{HCV}$ replicon abolished this difference, thus demonstrating that $\mathrm{HCV}$ induces Fth up-regulation at a transcriptional level.

To assess whether HCV NS proteins-induced Fth up-regulation is mediated by oxidative stress, we measured Fth mRNA levels in response to the antioxidant $\mathrm{N}$-acetyl cysteine (NAC). As expected, HCV replicon expression increased ROS production (Figure 5B). Interestingly, NAC treatment, while lowering ROS production, did not influence $\mathrm{HCV}$-induced Fth up-regulation (Figure $5 \mathrm{~B}$ and $\mathrm{C}$ ). Similar results were obtained by using L-ascorbic acid as an antioxidant (data not shown). These results demonstrate that $\mathrm{HCV}$ replicon expression leads to Fth transcriptional activation independent of cellular oxidative stress status. 
A

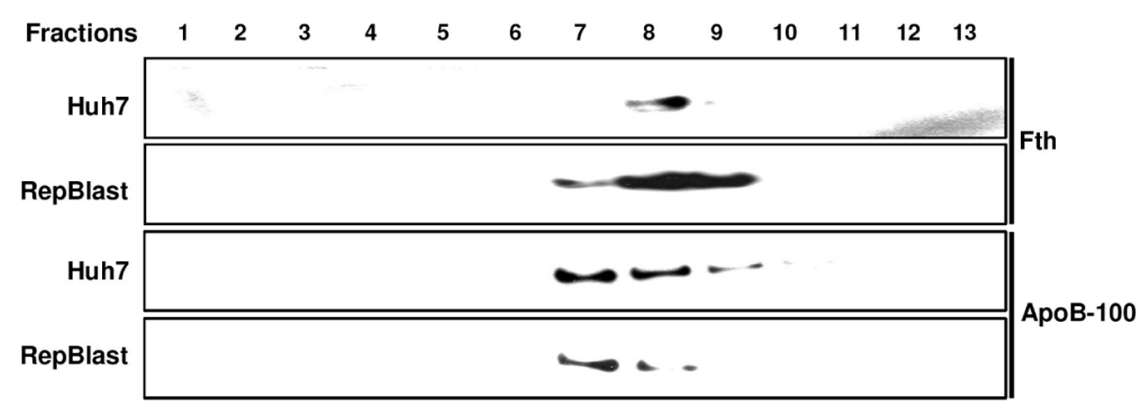

B

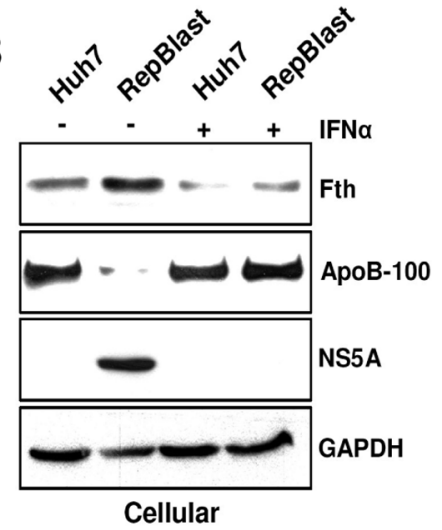

C

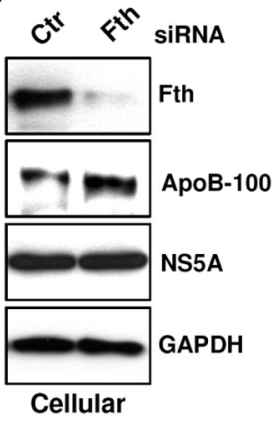

D

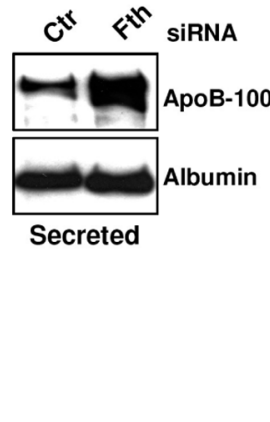

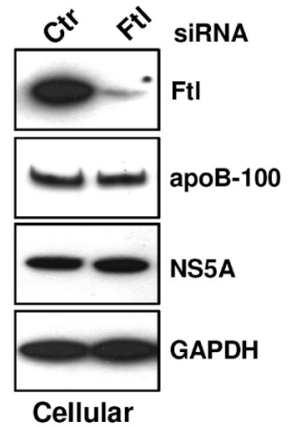

E
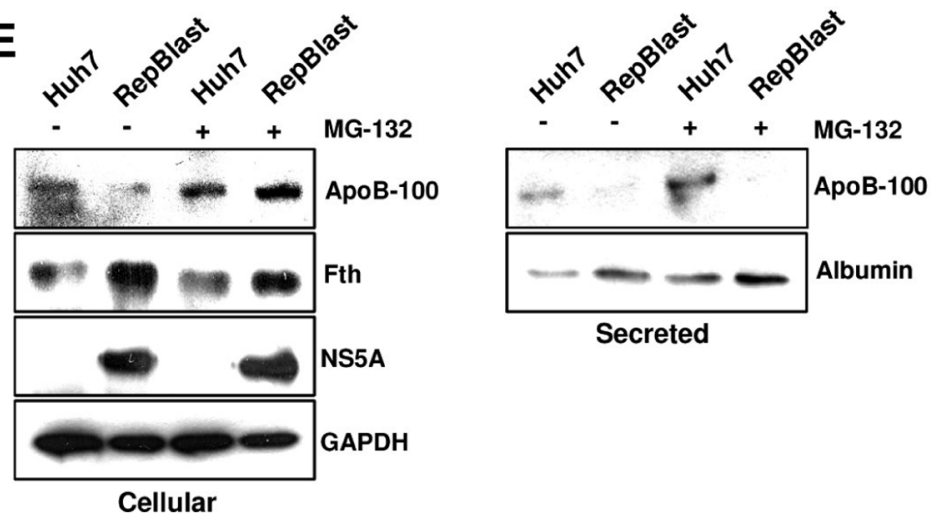

Secreted

Figure 4. HCV-mediated inhibition of apoB-100 secretion occurs by Fth up-regulation. (A) Fth and apoB-100 Western blot analysis of membrane floatation-derived vesicles in the indicated cell lines. (B) Fth and apoB-100 Western blot analysis of total protein extracts isolated from the indicated cell lines in the presence or absence of IFN $\alpha$. (C) Cellular and secreted Western blot analysis for the indicated proteins in RepBlast cells transfected either with Fth specific or control siRNA. (D) Cellular and secreted Western blot analysis for the indicated proteins in RepBlast cells transfected either with Ftl specific or control siRNA. (E) Fth and apoB-100 Western blot analysis in total protein extracts and secreted proteins obtained from the indicated cell lines in the presence or absence of MG-132. NS5A was used as control for HCV-replicon expression for all the analyses; GAPDH and albumin for protein loading control.

Viral Protein NS5A Is Sufficient to Up-regulate Ferritin Heavy Chain Expression

NS5A protein was found to physically interact with Fth. ${ }^{21}$ To investigate the role of NS5A in the Fth-mediated inhibition of apoB-100 production, we generated Huh7 cells that only expressed this viral protein. As shown in Figure 6A, NS5A was found to be sufficient in inducing $F$ th up-regulation and decreasing intracellular and secreted apoB-100 levels. Moreover, similar to HCV-replicon harboring cells, the siRNAmediated down-regulation of Fth protein induced increased intracellular and secreted amounts of apoB-100 in Huh7 cells expressing NS5A alone (Figure 6B).

Taken together, these results demonstrate that the viral protein NS5A is sufficient to induce the synthesis of Fth, which acts as determinant for HCV-induced inhibition of apoB-100 production.

\section{Fth Is Required for Robust HCV Infections}

Next, we aimed at extending our observations to the JFH-1 $\mathrm{HCV} c \mathrm{c}$ (genotype $2 \mathrm{a}$ ) natural infection system which generates infectious HCV particles in the Huh7-5.1 hepatoma cell line, 
A

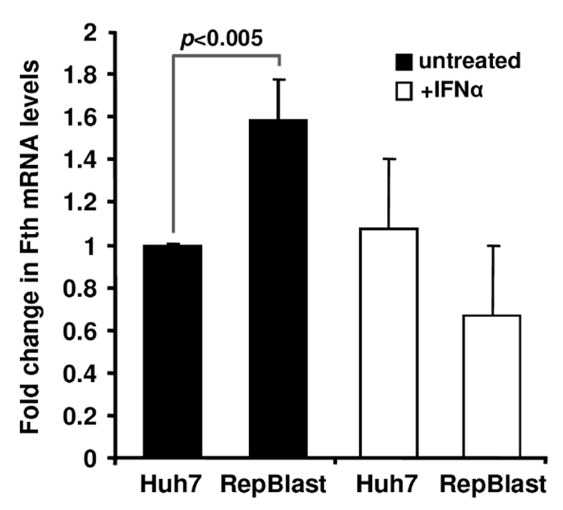

C
B

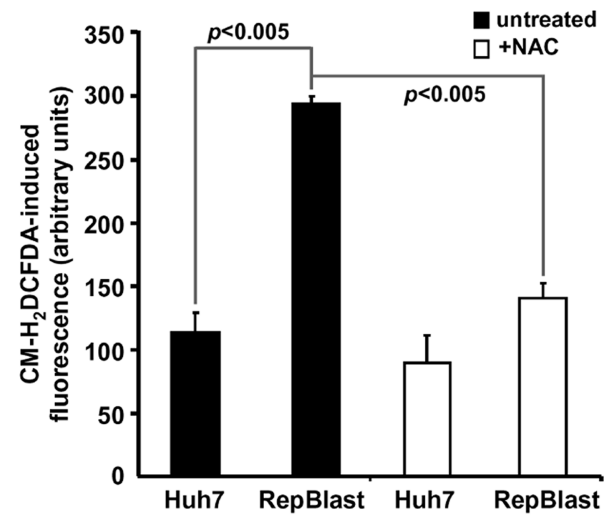

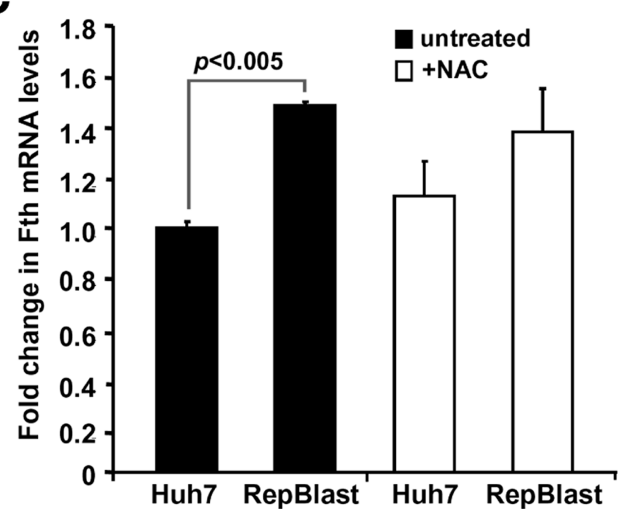

Figure 5. HCV replicon expression transcriptionally activates Fth independent of oxidative stress. (A) Fth RT-qPCR analysis in the indicated cell lines in the presence or absence of IFN $\alpha$. (B) CM- ${ }_{2}$ DCFDA (5-(and-6)-chloromethyl-2',7'-dichlorodihydrofluorescein diacetate, acetyl ester)induced fluorescence flow cytometry assay and (C) Fth RT-qPCR analysis in the indicated cell lines in the presence or absence of N-acetyl cysteine (NAC) $(10 \mu \mathrm{M}$ for $4 \mathrm{~h})$. Data represent means $\pm \mathrm{SD}$ from biological triplicates.

A

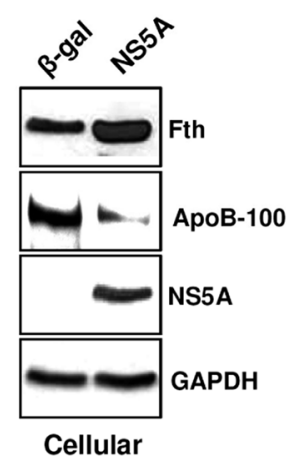

B

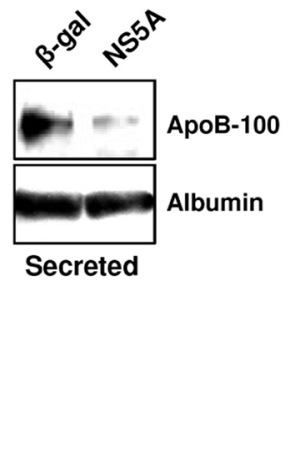

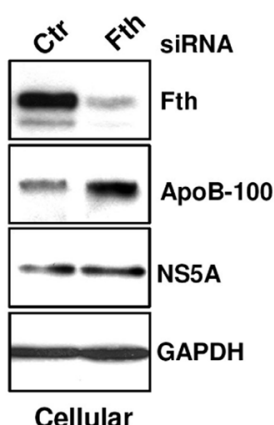

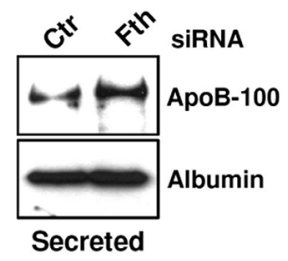

Secreted

Figure 6. NS5A alone recapitulates the HCV-induced inhibition of apoB-100 secretion. (A) Cellular and secreted Western blot analysis for the indicated proteins in the presence or absence of NS5A. Huh7 cells were infected with NS5A or $\beta$-gal expressing vectors. (B) Cellular and secreted Western blot analysis for the indicated proteins in NS5A-infected Huh7 cells transfected either with Fth specific or control siRNA. GAPDH and albumin were used for protein loading control.

thus recapitulating the complete viral life cycle. ${ }^{22}$ To this end, naive Huh7.5.1 cells were HCV-infected and at 4 days postinfection, both the apoB-100 production and the Fth expression were measured by Western blotting analysis. As shown in Figure 7A, Fth expression in HCV-infected cells (JFH-1) was found to be up-regulated while the intracellular and secreted apoB-100 levels were affected. Since HCV production is dependent on assembly and secretion of very low-density lipoproteins ${ }^{13}$ we assessed whether Fth downregulation, restoring high levels of apoB-100 production, may boost viral production. To this aim, Huh7-5.1 cells were transfected with either Fth-specific siRNA or control siRNA and, after $24 \mathrm{~h}$, infected with HCV $(\mathrm{MOI}=0.1)$ for $2 \mathrm{~h}$. The effect of Fth siRNA on HCV production was assessed by quantifying the viral RNA released in the medium 4 days postinfection. Surprisingly, while apoB-100 cellular and secreted levels were found to be increased, Fth specific down-regulation considerably reduced the viral RNA in the medium (Figure $7 \mathrm{D}$ ). These data indicate that $\mathrm{Fth}$ up-regulation is required for efficient viral infection as underscored by the inverse correlation found between the intracellular amounts of $\mathrm{HCV}$ core protein and Fth expression (Figure $7 \mathrm{~B}$ and $\mathrm{C}$ ). 
A

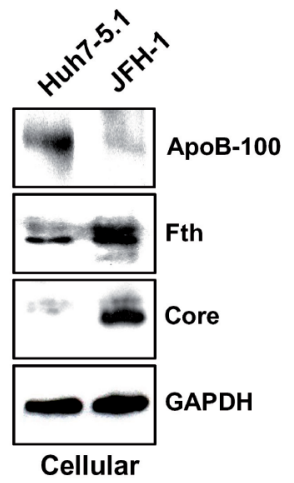

C

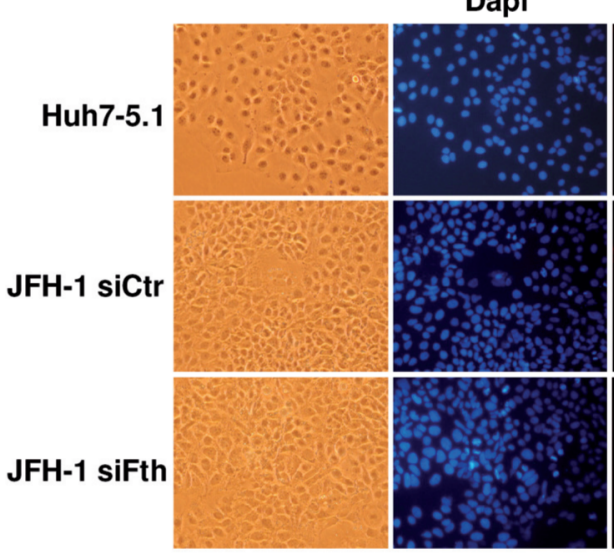

D

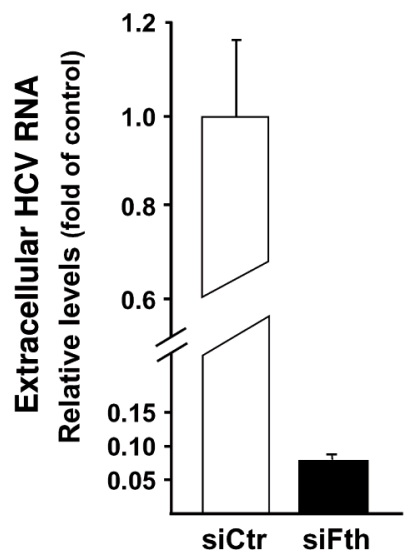

B

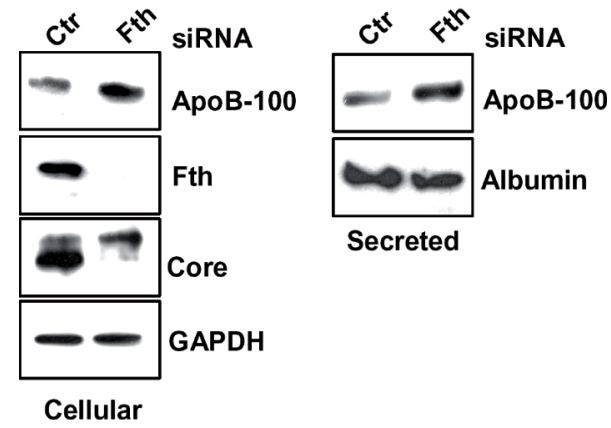

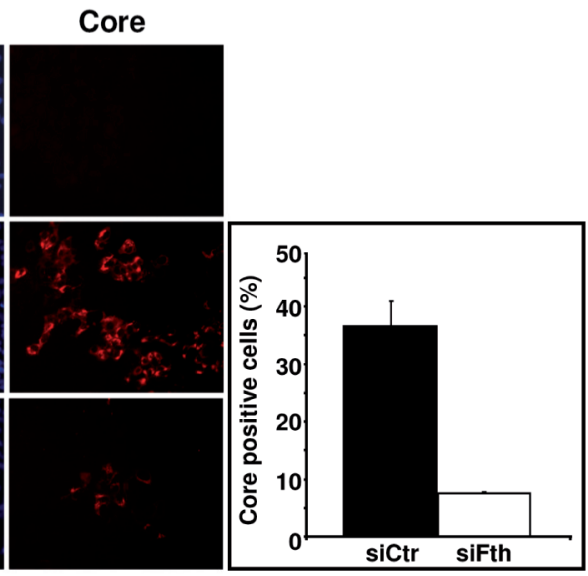

$\mathbf{E}$

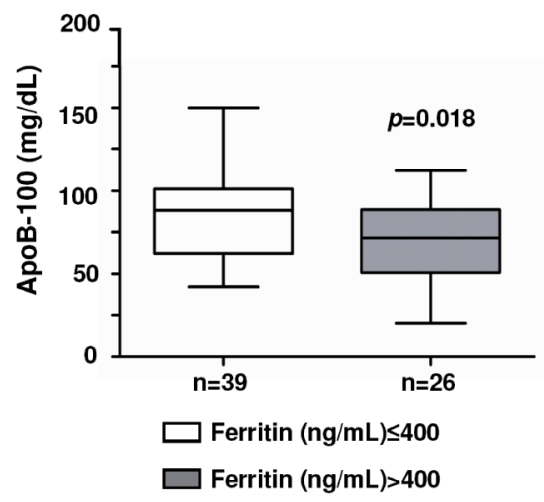

Figure 7. ApoB-100 secretion inversely correlates with ferritin concentration in HCV-infected cells and patients. (A) Cellular and secreted Western blot analysis for the indicated proteins in parental (Huh7-5.1) and JFH-1-infected cells. Core was used as control for HCV infection; GAPDH and albumin for protein loading control. (B) Cellular and secreted Western blot analysis for the indicated proteins in JFH-1-infected cells transfected either with Fth specific or control siRNA. Cells were transfected $24 \mathrm{~h}$ before infection and lysed 4 days postinfection. (C) Immunofluorescence analysis for core expression in JFH-1-infected cells transfected either with Fth specific or control siRNA $24 \mathrm{~h}$ before infection. Cells were transfected $24 \mathrm{~h}$ before infection, fixed 4 days postinfection and subjected to immunofluorescence microscopy as described in the Experimental section. The histogram in the box indicates the percentage of core positive cells (means \pm SD; $p<0.0005$ ) obtained from counts of 7 different random fields. (D) RT-qPCR analysis of HCV RNA copy number present in the culture medium of Fth specific or control siRNA-treated cells 4 days after infection. Data represent means \pm SD from biological triplicates. (E) Analysis of circulating apoB-100 levels in HCV-infected patients with normal $(\leq 400 \mathrm{ng} / \mathrm{mL})$ and high $(>400 \mathrm{ng} / \mathrm{mL})$ plasma ferritin concentrations. Changes in circulating apoB-100 levels are represented as box plots. The number of patients $(n)$ is indicated in the horizontal axes. For details and statistical analysis, see Supporting Information Table 2.

Inverse Correlation between Fth Expression and apoB-100 Production in HCV-infected Patients

Finally, we verified the robustness of the inverse association between Fth expression and apoB-100 secretion in HCV-infected patients. Hepatic iron accumulation has been shown to be present mainly in male patients with chronic hepatitis C. ${ }^{23}$ However, it remains unknown whether high liver ferritin concentration could be related to apoB-100 dysregulation. 
We addressed this issue analyzing the relationship between $\mathrm{HCV}$ infection, circulating apoB-100 levels and plasma ferritin concentration; the latter is in fact known to reflect the level of ferritin chain expression in the liver. ${ }^{24}$ As expected, high ferritin concentration $(>400 \mathrm{ng} / \mathrm{mL})$ (found exclusively in male donors) represented $40 \%$ of the $\mathrm{HCV}$-infected patients (Figure $7 \mathrm{E}$ and Supporting Information Table 2). Interestingly, we found the mean value of apoB-100 concentration in patients with high ferritin levels to be significantly lower $(70 \pm 23 \mathrm{mg} / \mathrm{dL}, p=$ 0.018 ) than what was observed in the group with normal ferritin levels $(86 \pm 27 \mathrm{mg} / \mathrm{dL})$, thus confirming the inverse association between Fth expression and the levels of apoB-100 secretion. Comparable results were obtained considering only the male population in the low ferritin group (Supporting Information Table 3).

Altogether, our results demonstrate that Fth up-regulation is the mechanism by which HCV affects apoB-100 production.

\section{DISCUSSION}

Here we have demonstrated that HCV infection up-regulates Fth expression which in turn specifically affects the production of apoB-100-containing lipoprotein particles. Our contribution originated from proteomic analysis and a literature oriented hypothesis. In fact, two elegant reports demonstrated the influences of: (i) $\mathrm{HCV}$ on hepatic iron accumulation ${ }^{25}$ and (ii) Fth up-regulation on apoB-100 secretion. ${ }^{18}$ We linked these observations to new in vivo and in vitro findings.

Specifically, Nishina and colleagues demonstrated that in transgenic mice, the $\mathrm{HCV}$-induced down-regulation of hepatic hepcidin leads to hepatic iron overloading and consequently, to ferritin up-regulation. ${ }^{25}$ Here, we show that HCV-induced Fth up-regulation also occurs at a transcriptional level, independent of an oxidative stress-based mechanism.

Hevi and Chuck clarified the intersection between the pathways for iron storage and apoB-100 production: Fth was described as apoB-100 binding protein, inhibiting its secretion. ${ }^{18}$ Here, we show that the viral protein NS5A alone can sufficiently increase $F$ th expression leading to reduced levels of apoB-100 secretion.

Although HCV and apoB-100-containing lipoprotein assembly machineries share the same cellular compartments ${ }^{26}$ and NS5A physically interacts with apoB-100, ${ }^{12}$ our finding excludes the hypothesis that the viral protein has an exclusive role in blocking exportation of apoB-100. In fact, a specific down-regulation of Fth by siRNA restores high levels of apoB-100 production, even in the presence of NS5A.

In search of the molecular mechanism causing $\mathrm{HCV}$-induced Fth-mediated inhibition of apoB-100 production, we highlighted that HCV-induced Fth expression blocks apoB-100 secretion, leading to increased degradation via proteasomes. In light of this, the apoB-100 intracellular decreased levels are not the result of a general apoB-100 synthesis down-regulation but rather the result of post-translational degradation. So far, the paucity of available informations on VLDL biogenesis, renders investigation of the molecular platform blocking ER apoB-100 export difficult; further investigations on ER-associated vesicle packaging and release of apoB-100 are needed.

The HCV-induced decrement of apoB-100-containing lipoprotein secretion is in contrast with the functional role of the VLDL pathway in the assembly and secretion of infectious viral particles. ${ }^{13}$ The observation that high levels of Fth expression are required for efficient viral infection might explain this paradox. In fact, in cells receiving the Fth siRNA, potential increments of viral particle production resulting from the increased apoB-100 secretion were nullified from a reduced level of infection. Moreover, since Fth down-regulation do not affect $\mathrm{HCV}$ replication (Figure 4C), the reduced levels of intracellular core expression observed in $\mathrm{HCV}$-infected cells (Figure 7B and C) suggest a potential involvement of Fth in the first steps of viral life cycle (e.g., cell attachment and entry). In this scenario, HCV infection might benefit from the Fth binding affinity for various molecules and several of its nonironmediated functions. ${ }^{27}$ Future experiments on topology and processes involving the interactions among Fth, HCV envelope proteins and viral cell surface receptors might unveil a possible role of Fth in the HCV life cycle.

To our knowledge, our in vivo data are the first reports that show the inverse correlation between plasma apoB-100 and ferritin concentrations. The novelty of our finding is due to the paucity of apoB-100 assays performed in the monitoring of lipid metabolism, both in the clinical practice of $\mathrm{HCV}$-infection and in the treatment of iron-related diseases.

While supported by in vitro observations, the in vivo results remain to be integrated and interpreted in a more general context. Indeed, a variety of factors as hormones, chemokines, cytokines, diet, life style, genetic factors, viral load and degree of liver damage are known to influence serum apoB-100 and ferritin concentrations. ${ }^{2,9,11,20,28}$

However, we believe that our results provide a new rationale for iron depletion therapy in $\mathrm{HCV}$-infected patients within the framework of interferon-based therapy. ${ }^{29,30}$ In fact, iron depletion, which reduces hepatic ferritin concentration and favors lipid exportation, might be taken into account as an additional therapeutic strategy to counteract hepatic lipid accumulation and hepatitis $\mathrm{C}$ progression. Moreover, iron accumulation is also a serious risk factor for HCC, playing a critical role in the onset of liver steatosis ${ }^{31}$ by both increasing lipidogenesis and reducing intracellular lipid catabolism. ${ }^{32,33}$

In conclusion, our study indicates that Fth is an important regulator of apoB-100-containing lipoproteins production and a host factor required for $\mathrm{HCV}$ infection, thus allowing to hypothesize new therapeutic interventions.

\section{ASSOCIATED CONTENT}

\section{Supporting Information}

Supplemental data as described in the text. This material is available free of charge via the Internet at http://pubs.acs.org.

\section{AUTHOR INFORMATION}

\section{Corresponding Author}

*Department of Cellular Biotechnologies and Haematology, Sapienza University of Rome, Via Regina Elena 324, 00161, Rome, Italy. Telephone: (+39) 06 49918246/7/8. Fax number: (+39) 060644252865 . E-mail: tripodi@bce.uniroma1.it.

\section{Author Contributions}

These authors contributed equally to the work.

Notes

The authors declare no competing financial interest.

\section{ACKNOWLEDGMENTS}

We are grateful to Dr. Marco Corazzari for his technical assistance and to Dr. G. M. Fimia for pCLBCX-NS5A retroviral construct. We are deeply grateful to Ms. Andrea Baker (INMI Rome, Italy) for the editing. 
This work was supported by grants from the Italian Ministry of Health (Ministero della Salute Ricerca Finalizzata Onc. Ord. 35/07, Convenzione N. 40H27, Ricerca Corrente), the Ministry of the University and Scientific Research (MIUR Ministero dell'Università e Ricerca Scientifica) and the Italian Association for Cancer Research (Associazione Italiana per la Ricerca sul Cancro (AIRC).

\section{REFERENCES}

(1) National Institutes of Health Consensus Development Conference. Management of hepatitis C. Hepatology 2002, 36, No. S1e252.

(2) Petit, J. M.; Benichou, M.; Duvillard, L.; Jooste, V.; Bour, J. B.; Minello, A.; Verges, B.; Brun, J. M.; Gambert, P.; Hillon, P. Hepatitis C virus-associated hypobetalipoproteinemia is correlated with plasma viral load, steatosis, and liver fibrosis. Am. J. Gastroenterol. 2003, 98, $1150-54$.

(3) Negro, F. Abnormalities of lipid metabolism in hepatitis C virus infection. Gut 2010, 59, 1590-91.

(4) Serfaty, L.; Andreani, T.; Giral, P.; Carbonell, N.; Chazouillères, O.; Poupon, R. Hepatitis $\mathrm{C}$ virus induced hypobetalipoproteinemia: a possible mechanism for steatosis in chronic hepatitis C. J. Hepatol. 2001, 34, 428-34.

(5) Tarugi, P.; Averna, M.; Di Leo, E.; Cefalù, A. B.; Noto, D.; Magnolo, L.; Cattin, L.; Bertolini, S.; Calandra, S. Molecular diagnosis of hypobetalipoproteinemia: an ENID review. Atherosclerosis 2007, 195, e19-27.

(6) Olofsson, S. O.; Stillemark-Billton, P.; Asp, L. Intracellular assembly of VLDL: two major steps in separate cell compartments. Trends Cardiovasc. Med. 2000, 10, 338-45.

(7) Fisher, E. A.; Ginsberg, H. N. Complexity in the secretory pathway: the assembly and secretion of apolipoprotein B-containing lipoproteins. J. Biol. Chem. 2002, 277, 17377-80.

(8) Raabe, M.; Véniant, M. M.; Sullivan, M. A.; Zlot, C. H.; Björkegren, J.; Nielsen, L. B.; Wong, J. S.; Hamilton, R. L.; Young, S. $\mathrm{G}$. Analysis of the role of microsomal triglyceride transfer protein in the liver of tissue-specific knockout mice. J. Clin. Invest. 1999, 103, 1287-98.

(9) Sharp, D.; Blinderman, L.; Combs, K. A.; Kienzle, B.; Ricci, B.; Wager-Smith, K.; Gil, C. M.; Turck, C. W.; Bouma, M. E.; Rader, D. J.; Aggerbeck, L. P.; Gregg, R. E.; Gordon, D. A.; Wetterau, J. R. Cloning and gene defects in microsomal triglyceride transfer protein associated with abetalipoproteinemia. Nature 1993, 365, 65-9.

(10) Fisher, E. A.; Zhou, M.; Mitchell, D. M.; Wu, X.; Omura, S.; Wang, H.; Goldberg, A. L.; Ginsberg, H. N. The degradation of apolipoprotein $\mathrm{B} 100$ is mediated by the ubiquitin proteasome pathway and involves heat shock protein 70. J. Biol. Chem. 1997, 272, 20427-34.

(11) Young, S. G.; Hubl, S. T.; Smith, R. S.; Snyder, S. M.; Terdiman, J. F. Familial hypobetalipoproteinemia caused by a mutation in the apolipoprotein $B$ gene that results in a truncated species of apolipoprotein B (B-31). A unique mutation that helps to define the portion of the apolipoprotein $\mathrm{B}$ molecule required for the formation of buoyant, triglyceride-rich lipoproteins. J. Clin. Invest. 1990, 85, 933-42.

(12) Domitrovich, A. M.; Felmlee, D. J.; Siddiqui, A. Hepatitis C virus nonstructural proteins inhibit apolipoprotein B100 secretion. J. Biol. Chem. 2005, 280, 39802-8.

(13) Huang, H.; Sun, F.; Owen, D. M.; Li, W.; Chen, Y.; Gale, M; Jr., $\mathrm{Ye}, \mathrm{J}$. Hepatitis $\mathrm{C}$ virus production by human hepatocytes dependent on assembly and secretion of very low-density lipoproteins. Proc. Natl. Acad. Sci. U.S.A. 2007, 104, 5848-53.

(14) Mancone, C.; Steindler, C.; Santangelo, L.; Simonte, G.; Vlassi, C.; Longo, M. A.; D’Offizi, G.; Di Giacomo, C.; Pucillo, L. P.; Amicone, L.; Tripodi, M.; Alonzi, T. Hepatitis C virus production requires apolipoprotein A-I and affects its association with nascent low-density lipoproteins. Gut 2011, 60, 378-86.
(15) Diamond, D. L.; Jacobs, J. M.; Paeper, B.; Proll, S. C.; Gritsenko, M. A.; Carithers, R. L. Jr.; Larson, A. M.; Yeh, M. M.; Camp, D. G. 2nd; Smith, R. D.; Katze, M. G. Proteomic profiling of human liver biopsies: hepatitis $\mathrm{C}$ virus-induced fibrosis and mitochondrial dysfunction. Hepatology 2007, 46, 649-57.

(16) Rashid, K. A.; Hevi, S.; Chen, Y.; Le Cahérec, F.; Chuck, S. L. A proteomic approach identifies proteins in hepatocytes that bind nascent apolipoprotein B. J. Biol. Chem. 2002, 277, 22010-7.

(17) Seki, T.; Kunichika, T.; Watanabe, K.; Orino, K. Apolipoprotein $\mathrm{B}$ binds ferritin by hemin-mediated binding: evidence of direct binding of apolipoprotein B and ferritin to hemin. Biometals 2008, 21, 61-9.

(18) Hevi, S.; Chuck, S. L. Ferritins can regulate the secretion of apolipoprotein B. J. Biol. Chem. 2003, 278, 31924-9.

(19) Kwak, E. L.; Larochelle, D. A.; Beaumont, C.; Torti, S. V.; Torti, F. M. Role for NF-kappa B in the regulation of ferritin $\mathrm{H}$ by tumor necrosis factor-alpha. J. Biol. Chem. 1995, 270, 15285-93.

(20) Torti, F. M.; Torti, S. V. Regulation of ferritin genes and protein. Blood 2002, 99, 3505-16.

(21) Ahn, J.; Chung, K. S.; Kim, D. U.; Won, M.; Kim, L.; Kim, K. S.; Nam, M.; Choi, S. J.; Kim, H. C.; Yoon, M.; Chae, S. K.; Hoe, K. L. Systematic identification of hepatocellular proteins interacting with NS5A of the hepatitis C virus. J. Biochem. Mol. Biol. 2004, 37, 741-8.

(22) Wakita, T.; Pietschmann, T.; Kato, T.; Date, T.; Miyamoto, M.; Zhao, Z.; Murthy, K.; Habermann, A.; Kräusslich, H. G.; Mizokami, M.; Bartenschlager, R.; Liang, T. J. Production of infectious hepatitis C virus in tissue culture from a cloned viral genome. Nat. Med. 2005, 11, 791-6.

(23) Farinati, F.; Cardin, R.; De Maria, N.; Della Libera, G.; Marafin, $\mathrm{C}$. Iron storage, lipid peroxidation and glutathione turnover in chronic anti-HCV positive hepatitis. J. Hepatol. 1995, 22, 449-56.

(24) Finch, C. A.; Bellotti, V.; Stray, S.; Lipschitz, D. A.; Cook, J. D.; Pippard, M. J.; Huebers, H. A. Plasma ferritin determination as a diagnostic tool. West J. Med. 1986, 145, 657-63.

(25) Nishina, S.; Korenaga, M.; Hidaka, I.; Shinozaki, A.; Sakai, A.; Gondo, T.; Tabuchi, M.; Kishi, F.; Hino, K. Hepatitis C virus-induced reactive oxygen species raise hepatic iron level in mice by reducing hepcidin transcription. Gastroenterology 2008, 134, 226-38.

(26) Gastaminza, P.; Cheng, G.; Wieland, S.; Zhong, J.; Liao, W.; Chisari, F. V. Cellular determinants of hepatitis C virus assembly, maturation, degradation, and secretion. J. Virol. 2008, 82, 2120-9.

(27) Arosio, P.; Ingrassia, R.; Cavadini, P. Ferritins: a family of molecules for iron storage, antioxidation and more. Biochim. Biophys. Acta 2009, 1790, 589-99.

(28) Clément, S.; Juge-Aubry, C.; Sgroi, A.; Conzelmann, S.; Pazienza, V.; Pittet-Cuenod, B.; Meier, C. A.; Negro, F. Monocyte chemoattractant protein-1 secreted by adipose tissue induces direct lipid accumulation in hepatocytes. Hepatology 2008, 48, 799-807.

(29) Fontana, R. J.; Israel, J.; LeClair, P.; Banner, B. F.; Tortorelli, K. Iron reduction before and during interferon therapy of chronic hepatitis C: results of a multicenter, randomized, controlled trial. Hepatology 2000, 31, 730-6.

(30) Kaito, M.; Iwasa, M.; Kobayashi, Y.; Fujita, N.; Tanaka, H.; Gabazza, E. C.; Adachi, Y.; Kojima, Y.; Nakagawa, N.; Watanabe, S. Iron reduction therapy by phlebotomy reduces lipid peroxidation and oxidative stress in patients with chronic hepatitis C. J. Gastroenterol. 2006, 41, 921-2.

(31) Furutani, T.; Hino, K.; Okuda, M.; Gondo, T.; Nishina, S.; Kitase, A.; Korenaga, M.; Xiao, S. Y.; Weinman, S. A.; Lemon, S. M.; Sakaida, I.; Okita, K. Hepatic iron overload induces hepatocellular carcinoma in transgenic mice expressing the hepatitis $\mathrm{C}$ virus polyprotein. Gastroenterology 2006, 130, 2087-98.

(32) Graham, R. M.; Chua, A. C.; Carter, K. W.; Delima, R. D.; Johnstone, D.; Herbison, C. E.; Firth, M. J.; O’Leary, R.; Milward, E. A.; Olynyk, J. K.; Trinder, D. Hepatic iron loading in mice increases cholesterol biosynthesis. Hepatology 2010, 52, 462-71.

(33) Nishina, S.; Korenaga, M.; Hidaka, I.; Shinozaki, A.; Sakai, A.; Gondo, T.; Tabuchi, M.; Kishi, F.; Hino., K. Hepatitis C virus protein and iron overload induce hepatic steatosis through the unfolded protein response in mice. Liver Int. 2010, 30, 683-92. 